\title{
Autophagy activation promotes bevacizumab resistance in glioblastoma by suppressing Akt/mTOR signaling pathway
}

\author{
HE HUANG ${ }^{1}$, JIAN SONG ${ }^{2}$, ZHENG LIU $^{2}$, LI PAN $^{2}$ and GUOZHENG XU ${ }^{1}$ \\ ${ }^{1}$ Department of Neurosurgery, Southern Medical University, Guangzhou, Guangdong 510515; \\ ${ }^{2}$ Department of Neurosurgery, Wuhan General Hospital of PLA, Wuhan, Hubei 430070, P.R. China
}

Received March 29, 2017; Accepted October 5, 2017

DOI: $10.3892 / 01.2017 .7446$

\begin{abstract}
Glioblastomas are the most common primary and malignant brain tumors. The standard therapy includes surgery and radiotherapy plus chemotherapy, with additional bevacizumab to block the angiogenesis in tumors. However, the ever-growing tolerance of glioblastomas to chemotherapeutic drugs impairs the clinical outputs of tumor treatment. The present study investigated the tolerance of glioblastomas to bevacizumab. Although bevacizumab resulted in direct anti-proliferation and pro-apoptosis effects on glioblastoma cells via downregulating the anti-apoptotic proteins and upregulating the pro-apoptotic proteins, tolerance was also encountered that was mainly caused by autophagy induction in tumor cells. The suppressed Akt-mTOR signaling pathway led to the upregulated autophagy process. Blockade of the autophagy process significantly increased the tumor-suppressive effect of bevacizumab on glioblastoma cells. To our knowledge, the present study is the first to report the involvement of autophagy in the tolerance of glioblastomas to bevacizumab. Therefore, autophagy inhibition may be considered a novel way to overcome the tolerance of glioblastomas to anti-angiogenic agents.
\end{abstract}

\section{Introduction}

Malignant glioblastoma is an aggressive and incurable tumor, with an annual incidence of 5.26 per 100,000 population or 17,000 new diagnoses per year (1), which represents nearly $80 \%$ of diagnosed primary brain tumors. In children, glioblastoma accounts for about one-fifth of all childhood cancers (2). Glioblastoma is among the most feared types of cancers which are usually associated with poor prognosis and profoundly impaired life quality. Glioblastoma originates from glial cells

Correspondence to: $\mathrm{Dr}$ Guozheng $\mathrm{Xu}$, Department of Neurosurgery, Southern Medical University, 1838 North Guangzhou Avenue, Guangzhou, Guangdong 510515, P.R. China

E-mail: xuguozheng0314@163.com

Key words: glioblastoma, tolerance, bevacizumab, autophagy, Akt/mTOR signaling pathway in central nervous system, and previous work demonstrated that chromosome 10 loss, p16INK4a deletion, p14ARF, PTEN and p53 mutation, RB1 and MGMT methylation, EGFR amplification contributed to the pathogenesis of glioblastoma $(3,4)$. The current standard cure for newly diagnosed glioblastoma patients is surgical removal combined with radiotherapy and then chemotherapy with the temozolomide if the tumor is high-grade. However, the exact molecular cause of glioblastoma is hard to decipher. In addition, many glioblastoma patients show high resistances to these therapeutic treatments, especially for the standard chemo drugs-temozolomide and carmustine (BCNU), and thus tumor recurrences are frequent. For example, intensive studies found that the overexpression of MGMT (O6-methylguanine methyl transferase) and inactivation mutations in the mismatch repair gene MSH6 (mutS homolog 6) were closely related with glioblastoma recurrent post-temozolomide treatment $(5,6)$, and the resistance mechanisms should have equal effects for carmustine in that they shared the same alkylating effect of DNA (7). Therefore, clearly revealing the underlying mechanisms of chemo-drug tolerance is the most urgent issue of improving the therapies of glioblastoma.

As is known to all, rapidly-proliferated and metastatic tumor cells consume lots of nutrients through adequate blood supply, so anti-angiogenic therapy has become an important method in the treatment of many solid tumors. Glioblastomas is highly vascularized (8) and overexpresses vascular endothelial growth factor A (VEGF-A) that is responsible for the angiogenesis (9). As the first available anti-angiogenic drug, bevacizumab was granted accelerated approval by FDA in 2009 for the treatment of recurrent multiform glioblastoma. Bevacizumab is one kind of recombinant humanized monoclonal antibody that targets for VEGF-A and blocks its binding to VEGF receptor, which thus inhibits the angiogenesis in a variety of diseases, especially for cancers, such as colorectal cancer, lung cancer, cervical cancer, ovarian cancer and renal cell carcinoma (Avastin Prescribing Information; Genentech, Inc., December 2016). In preclinical experiments and early clinical trials, bevacizumab had some efficacies on prolonging progression-free survival, possibly improving quality of life and decreasing steroid usage. However, it did not show an overall-survival benefit in a late clinical trial of patients with glioblastoma $(10,11)$. Some studies were performed to explore the reason of low efficacy of bevacizumab for 
glioblastoma patients. Several mechanisms, including receptor tyrosine kinase c-Met upregulation, myeloid cell infiltration and stem cell accumulation, were identified to be associated with the resistance of glioblastomas to anti-angiogenic therapy $(12,13)$. In colorectal cancer cells, people found that the prolonged activation of autocrine VEGF signaling might contribute to the bevacizumab resistance (14). To improve the efficacy of bevacizumab, additional researches are still required to explore the mechanisms of resistance, other pro-angiogenic pathways and new combination strategies.

Autophagy is a highly conserved system responsible for the removal of damaged organelles or misfolded proteins by lysosomal degradation, which contributes to maintain intercellular homeostasis. Previous studies demonstrated that autophagy could play significant roles in antigen presentation, cell death, bacterial and viral infection $(15,16)$. Dysfunction of autophagy is associated with the pathogenesis of metabolic and neurodegenerative diseases, viral infection, muscle diseases, cancer, and hepatic inflammation (17-19). Autophagy process consists of a series of steps: i) The initiation of the isolated membrane; ii) cargo recognition and nucleation; iii) elongation of the isolated membrane; iv) enclosure of membrane structures and formation of autophagosome; and v) maturation and degradation of engulfed proteins (20). During autophagy, microtubule associated protein 1-light chain 3 (LC3, one homolog of ATG8) is firstly loaded onto the membrane by conjugating with phosphatidylethanolamine (POPE) in the membrane, which will modify the curvature of membrane and promote the maturation of autophagosome. Then, the cargo is loaded into the autophagosome by the interaction between the specific receptors on cargo proteins and LC3 on the autophagosome membrane, in which the first identified selective receptor is SQSTM1(p62) (21). After formation, autophagosome will fuse with the lysosome to digest the loaded cargo proteins (22). Previous studies found that autophagy could either support or suppress the tumor cell growth depending on the cell context (23). In normal tissues and cells, autophagy serves as a tumor-suppressive process (24). However, once the malignant phenotype has been established, autophagy is often harnessed to facilitate tumor cell survival under metabolic stresses caused by antitumor agents (25). It was also reported that autophagy could be induced in response to chemotherapeutics, promoting the formation of drug-tolerance and the impairment of tumor therapy (26-28). Therefore, targeting autophagy is an attractive and promising therapeutic strategy to potentiate the effects of chemotherapy and improve clinical outputs in the treatment of cancer patients (29).

Until now, there are no available reports about the autophagy involved in the tolerance of glioblastomas to bevacizumab. Here, we used a glioblastoma cell line, U87-MG cells, to systematically study the anti-proliferation and pro-apoptosis effects of bevacizumab on glioblastoma cells. We found that bevacizumab could induce the downregulate the anti-apoptotic proteins and upregulate the pro-apoptotic proteins in glioblastomas cells to promote their apoptosis. However, glioblastomas cells were able to enhance their autophagy to tolerant bevacizumab through attenuating Akt-mTOR signaling pathway, while blockade of the autophagy process by its inhibitor could significantly increase the tumor-suppressive effect of bevacizumab on glioblastomas.

\section{Materials and methods}

Cell culture and reagents. The human glioblastoma cell line, U87-MG was bought from ATCC and maintained in Dulbecco's modified Eagle's medium (DMEM) (Gibco; Thermo Fisher Scientific, Inc., Waltham, MA, USA) and supplemented with $10 \%$ fetal bovine serum at $37^{\circ} \mathrm{C}$ in a humidified $5 \% \mathrm{CO}_{2}$ incubator. Although one research published in Science Translational Medicine revealed that glioma cell line U87-MG from ATCC was likely to be a bona fide human glioblastoma cell line of unknown origin (30), there was a research also declared that studies of U87 still reflected brain-cancer biology and didn't need to be tossed out (31). So, we still used the U87-MG cell line to study the glioblastoma just like this research (32) Chloroquine (CQ) was obtained from Sigma-Aldrich; Merck KGaA (Darmstadt, Germany). Bevacizumab was obtained from Roche Diagnostics (Basel, Switzerland). Anti-Bim, anti-Bcl-2, anti-Bax, anti-survivin, anti-cleaved caspase-3, anti-cleaved caspase-8, anti-cleaved caspase-9, anti-PARP, anti-LC3B-I, anti-LC3B-II, anti-SQSTM1 (p62), anti-Akt, anti-p70S6K, anti-mTOR, anti-GAPDH, anti-p-Akt (T308), anti-p-Akt (S473), anti-p-p70S6K (T389) and anti-p-mTOR (S2448) antibodies were from Cell Signaling Technology, Inc. (Danvers, MA, USA). MTT kit was from Thermo Fisher Scientific, Inc. Annexin V/PI kit was from Nanjing KeyGen Biotech Co., Ltd. (Nanjing, China).

Cell proliferation measurements by MTT. Before experiments, U87-MG cells growing in logarithmic phase were digested with $0.25 \%$ Trypsin-EDTA and pipetted into single cells. Cells were carefully counted by TC20 ${ }^{\mathrm{TM}}$ Automated Cell Counter (Bio-Rad Laboratories, Inc., Hercules, CA, USA) and $5 \times 10^{3}$ cells in $100 \mu \mathrm{l}$ medium per well were seeded into 96-well plate supplemented with different concentrations $(0$, $0.5,1,2,4,8,16,32 \mathrm{mg} / \mathrm{ml})$ of bevacizumab. For each concentration, five repeated wells were prepared and a blank control group with culture medium only was also set, and then they were cultured in the incubator for 24 or $48 \mathrm{~h}$, respectively. After that, the cell viability was measured with MTT kit following the manufacturer's instructions. Briefly, the medium was removed and replaced by $100 \mu \mathrm{l}$ of fresh phenol red-free culture medium. $10 \mu \mathrm{l}(10 \%$ of the volume of the culture medium) MTT reagent was gently loaded into the medium in each well, and then cultured in the incubator at $37^{\circ} \mathrm{C}$ for $4 \mathrm{~h}$. $75 \mu \mathrm{l}$ of medium was removed from each well and then $50 \mu \mathrm{l}$ DMSO was added into each well and mixed thoroughly with the pipette. The 96 -well plate was then incubated at $37^{\circ} \mathrm{C}$ for $10 \mathrm{~min}$. Then the samples were mixed again and the optical density (OD) was measured at $540 \mathrm{~nm}$ for each well by a plate reader (EON; BioTek Instruments, Inc., Winooski, VT, USA).

Cell apoptosis measurements by Annexin V/PI. Cells for Annexin V-FITC/PI staining were harvested at the same time points and with the same methods mentioned above. However, to avoid the cell damage due to trypsinization, trypsin without EDTA was used to digest the cells. Then the cells were stained with Annexin V-FITC/PI following the manufacturer's instruction. Briefly, $2 \times 10^{5}$ U87-MG cells were pooled and washed twice with cold PBS, and then re-suspended in $500 \mu \mathrm{l}$ binding buffer. After that, $5 \mu \mathrm{l}$ Annexin V-FITC and $5 \mu \mathrm{l}$ propidium 

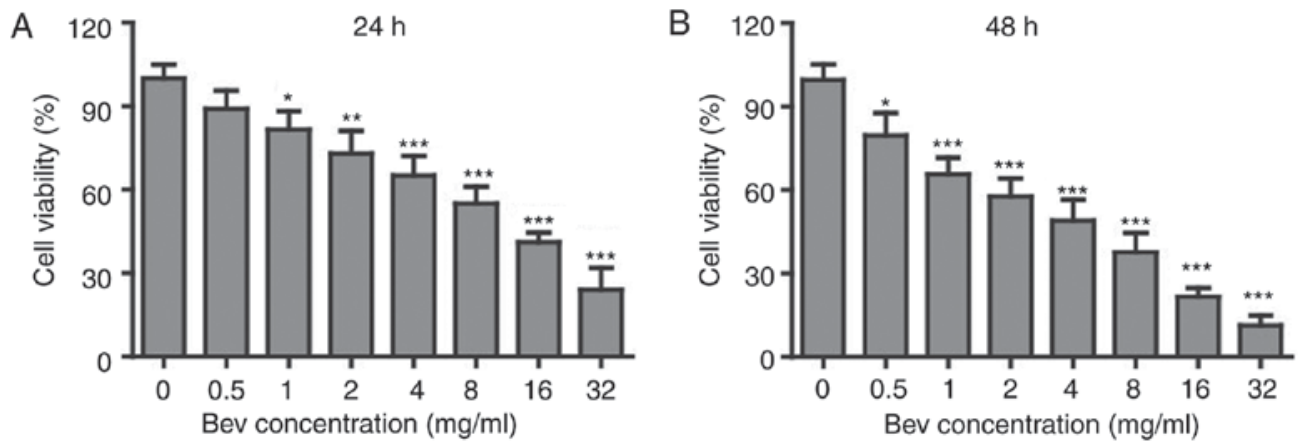

Figure 1. The anti-proliferation effect of bevacizumab on glioblastoma cells. U87-MG cells were treated with different concentrations of bevacizumab for $24 \mathrm{~h}(\mathrm{~A})$ and $48 \mathrm{~h}(\mathrm{~B})$, and then the cell viability was measured with MTT kit. Error bars represented mean \pm SD. P-values were determined by one-way ANOVA followed by Tukey's post hoc test. ${ }^{* * *} \mathrm{P}<0.001,{ }^{* * *} \mathrm{P}<0.01$, and ${ }^{*} \mathrm{P}<0.05$ vs. control group. ANOVA, analysis of variance.

iodide (PI) were added into the cell suspension and mixed equally by gently pipetting. Then the cell samples were incubated at room temperature for 5-15 min and protected from light during this process. Subsequently, the cell samples were analyzed by flow cytometry (FACSCalibur; BD Biosciences) to check the apoptosis within $1 \mathrm{~h}$. All experiments were performed in triplicate and repeated at least 3 times.

Protein extraction and western blot analysis. 5x10 $1078-\mathrm{MG}$ cells were seeded into 6-well plate supplemented with $5 \mathrm{ml}$ fresh DMEM medium and different concentrations of bevacizumab $(0,1,2,4 \mathrm{mg} / \mathrm{ml})$ for $48 \mathrm{~h}$. In the autophagy blocking experiment, $4 \mathrm{mg} / \mathrm{ml}$ bevacizumab with or without $10 \mu \mathrm{M}$ chloroquine were added into the medium for $48 \mathrm{~h}$. Then the cells were pooled and washed with PBS twice, then lysed by RIPA buffer. The same volume of cell lysates was mixed with $4 \mathrm{X}$ reducing loading buffer and then the mixtures were boiled for $10 \mathrm{~min}$. And the proteins were subjected for SDS-PAGE electrophoresis, and then the separated proteins in gel were transferred to PVDF membrane, which was subsequently blocked by $10 \%$ BSA and incubated with the indicated primary antibodies for the target proteins. After TBST washing for three times, the membrane was then incubated with the corresponding HRP-conjugated secondary antibodies. After TBST washing, the PVDF membrane was then incubated with ECL substrate and used for film exposure in dark room.

Data analysis. All experiments were performed at least for three times in triplicate, data were expressed as mean \pm standard deviation (SD). Statistical analyses were performed using GraphPad Prism 5 (GraphPad Software, Inc., La Jolla, CA, USA). Statistical significance was determined as indicated in the figure legends. $\mathrm{P}<0.05$ was considered significant. One-way analysis of variance (ANOVA) followed by Tukey's post hoc test was used to test for multiple comparison.

\section{Results}

Bevacizumab suppressed the proliferation of glioblastoma cells. In clinic, bevacizumab is used as an angiogenesis inhibitor. However, whether it can directly suppress the proliferation of tumor cells is not clear. To study the effects of bevacizumab on glioblastoma cells, we firstly administered U87-MG cells with various concentrations of bevacizumab for
24 and $48 \mathrm{~h}$ respectively, and then checked the cell proliferation and viability by MTT kit. To our surprise, the cell viability was getting lower down with the increasing concentration of bevacizumab (Fig. 1A), and the proliferation suppression was further enhanced if the treatment time was expanded for $48 \mathrm{~h}$ (Fig. 1B). When treated with $4 \mathrm{mg} / \mathrm{ml}$ bevacizumab for $48 \mathrm{~h}$, glioblastoma cells showed only about $50 \%$ of proliferation compared to those without bevacizumab treatment. According to this result, we mainly used 0, 1,2,4 mg/ml doses of bevacizumab for $48 \mathrm{~h}$ in later experiments. Taken together, this result demonstrated that bevacizumab could directly suppress the proliferation of glioblastoma cells in a dose and time dependent manner.

Bevacizumab promoted the apoptosis of glioblastoma cells. Besides the anti-proliferation effect, we also want to know whether bevacizumab can promote the apoptosis of glioblastoma cells. To address this question, we cultured U87-MG cells with various concentrations of bevacizumab for $48 \mathrm{~h}$, and then performed the cell apoptosis measurements using Annexin V/PI method. Without bevacizumab, U87-MG cells showed very low percentage of apoptosis (2.52 and 6.03\% for early and late stages of apoptosis, respectively). However, even low dose $(1 \mathrm{mg} / \mathrm{ml})$ of bevacizumab was able to induce significantly high level of apoptosis, both the early and late stages of apoptosis (15.97 and 11.14\%, respectively) (Fig. 2). More importantly, glioblastoma cells showed similar $(\sim 30 \%)$ apoptosis with 1 or $2 \mathrm{mg} / \mathrm{ml}$ concentrations of bevacizumab (Fig. 2B), that meant some glioblastoma cells could still survive under high dose of bevacizumab, which thus reflected that the tolerance of glioblastoma cells to bevacizumab occurred.

Bevacizumab downregulated anti-apoptotic protein level and augmented pro-apoptotic protein level of glioblastoma cells. To uncover the mechanism of bevacizumab on directly inhibiting proliferation and promoting apoptosis of tumor cells, we firstly treated U87-MG cells with different concentrations of bevacizumab for $48 \mathrm{~h}$, and then performed new biochemical experiments to assess the protein contents of Bcl-2 and survivin, which were typical anti-apoptotic markers. At the same time, we measured the expression levels of Bim, Bax and cleaved caspase $-3,-8$, and -9 which belonged to pro-apoptotic markers. PARP, which is involved in DNA damage repair in its full-length form, will be cleaved 

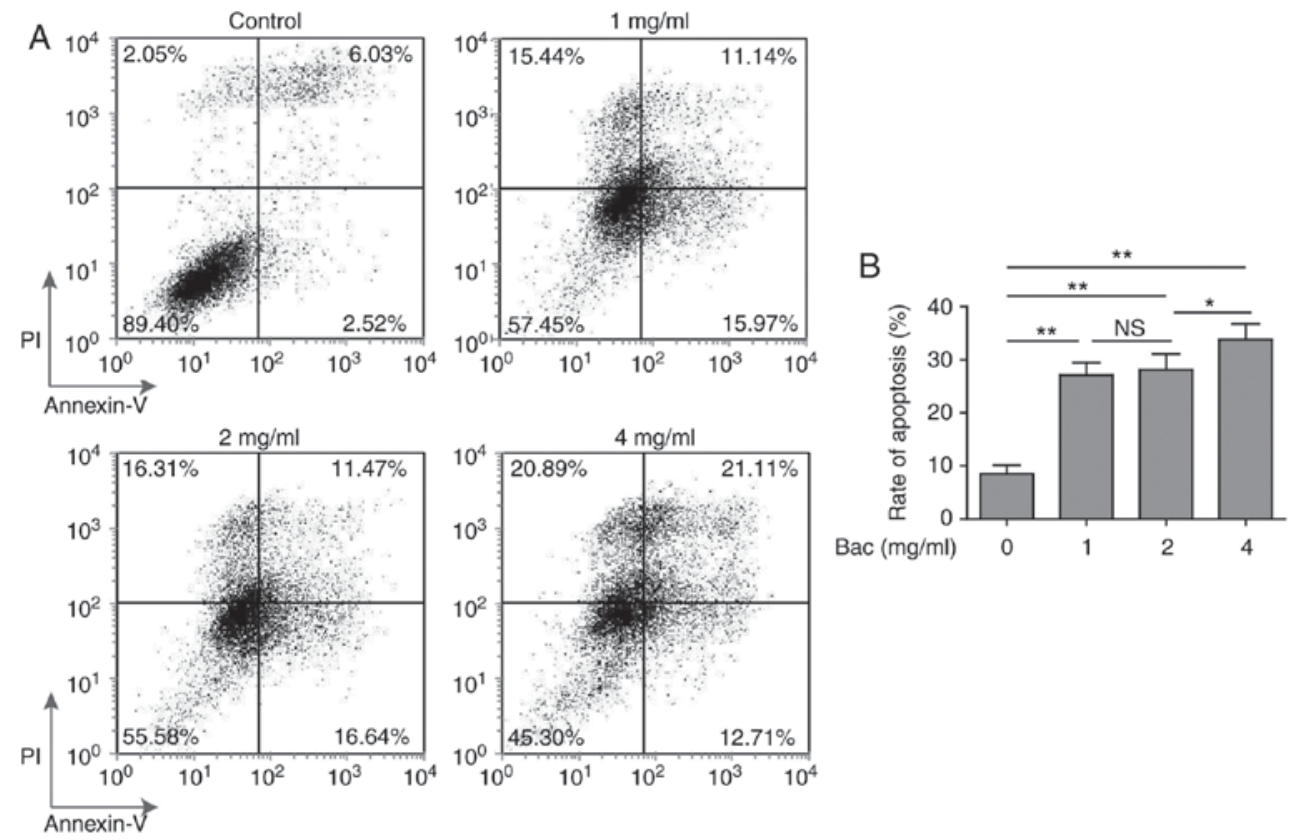

ac $(\mathrm{mg} / \mathrm{ml})$

Figure 2. The pro-apoptosis effect of bevacizumab on glioblastoma cells. U87-MG cells were treated with different concentrations of bevacizumab for $48 \mathrm{~h}$, and then the cell apoptosis was assessed by Annexin V/PI staining and flow cytometry analysis. (A) One representative FACS result from three independent experiments. (B) The rate of apoptosis for cells in A. Error bars represented mean \pm SD. P-values were determined by one-way ANOVA followed by Tukey's post hoc test. ${ }^{* *} \mathrm{P}<0.01,{ }^{*} \mathrm{P}<0.05$ and ns, not significant. ANOVA, analysis of variance.

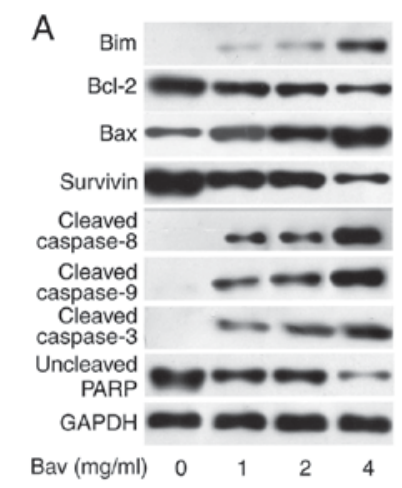

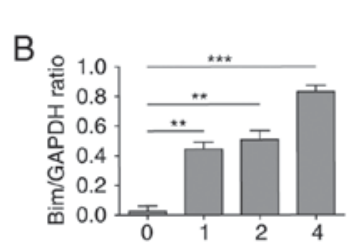

Bav concentration $(\mathrm{mg} / \mathrm{ml})$
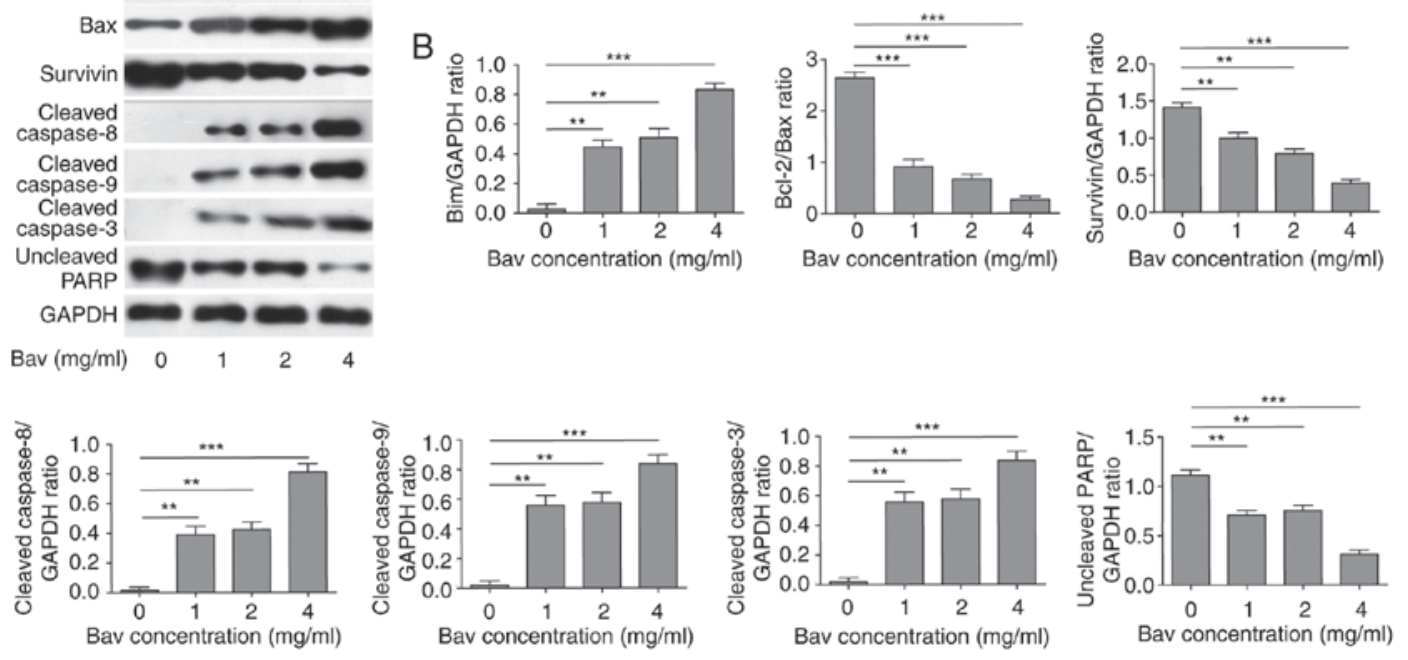

Figure 3. The attenuated anti-apoptotic protein level and augmented pro-apoptotic protein level of glioblastoma cells by bevacizumab treatment. (A) U87-MG cells were treated with different concentrations of bevacizumab for $48 \mathrm{~h}$, and then the anti-apoptotic protein contents of Bcl-2 and surviving, and the pro-apoptotic protein contents of Bim, Bax and cleaved caspase-3, -8 , and -9 , as well as the uncleaved PARP, were assessed by western blot analysis, respectively. GAPDH was used as the loading control. The result was a representative of three independent experiments. (B) The quantification of Bcl-2/Bax ratio and the expression levels of other proteins normalized to GAPDH for result in A. Error bars represented mean \pm SD. P-values were determined by one-way ANOVA followed by Tukey's post hoc test. ${ }^{* * * *} \mathrm{P}<0.001,{ }^{* *} \mathrm{P}<0.01$. ANOVA, analysis of variance.

into two parts by caspase-3, and the cleavage of PARP will hinder the DNA repair and therefore serves as an apoptosis marker. Herein we blotted the full length of PARP to reflect its cleavage status. The results clearly demonstrated that bevacizumab could significantly reduce the expression levels of anti-apoptotic proteins, including Bcl-2, survivin and full length of PARP (Fig. 3). Meanwhile, bevacizumab upregulated the expression levels of pro-apoptotic proteins, such as Bim,
Bax and cleaved caspase- $3,-8$ and -9 , which also promoted the cleavage of PARP (Fig. 3). Both mechanisms synergistically led to the decreased proliferation and increased apoptosis of glioblastoma cells with the treatment of bevacizumab.

Bevacizumab enhanced the autophagy of glioblastoma cells. In previous clinical application, there were obvious chemo-tolerance developed during glioblastoma treatment 

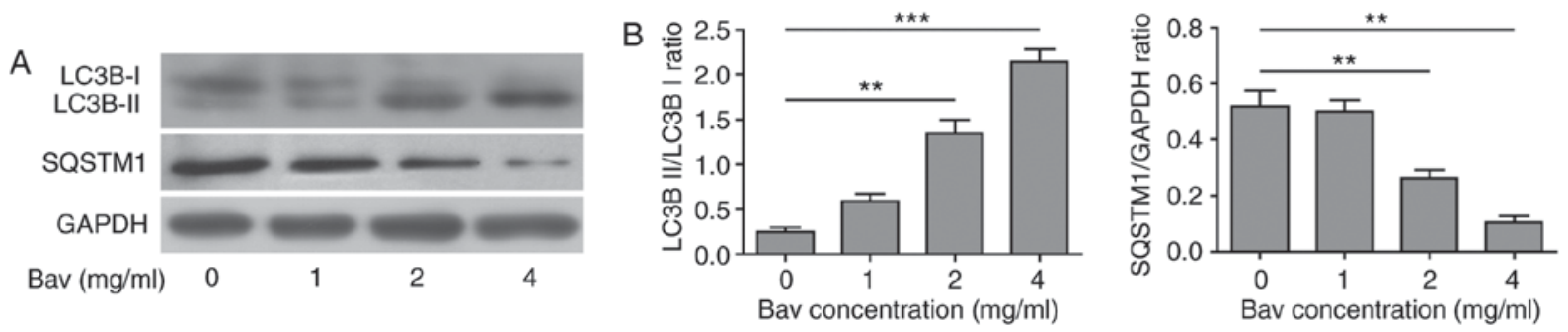

Figure 4. Enhanced autophagy of glioblastoma cells by bevacizumab treatment. (A) U87-MG cells were treated with different concentrations of bevacizumab for $48 \mathrm{~h}$, and then the cells were lysed for western blot analysis for indicative hallmarks of autophagy. GAPDH was used as the loading control. The result was a representative of three independent experiments. (B) The quantification of LC3B-II/LC3B-I ratio and SQSTM1(p62)/GAPDH ratio for result in A, both were taken as hallmarks to assess the autophagy level. Error bars represented mean \pm SD. P-values were determined by the one-way analysis of variance (ANOVA) followed by Tukey's post hoc test. ${ }^{* * *} \mathrm{P}<0.001,{ }^{* *} \mathrm{P}<0.01$.
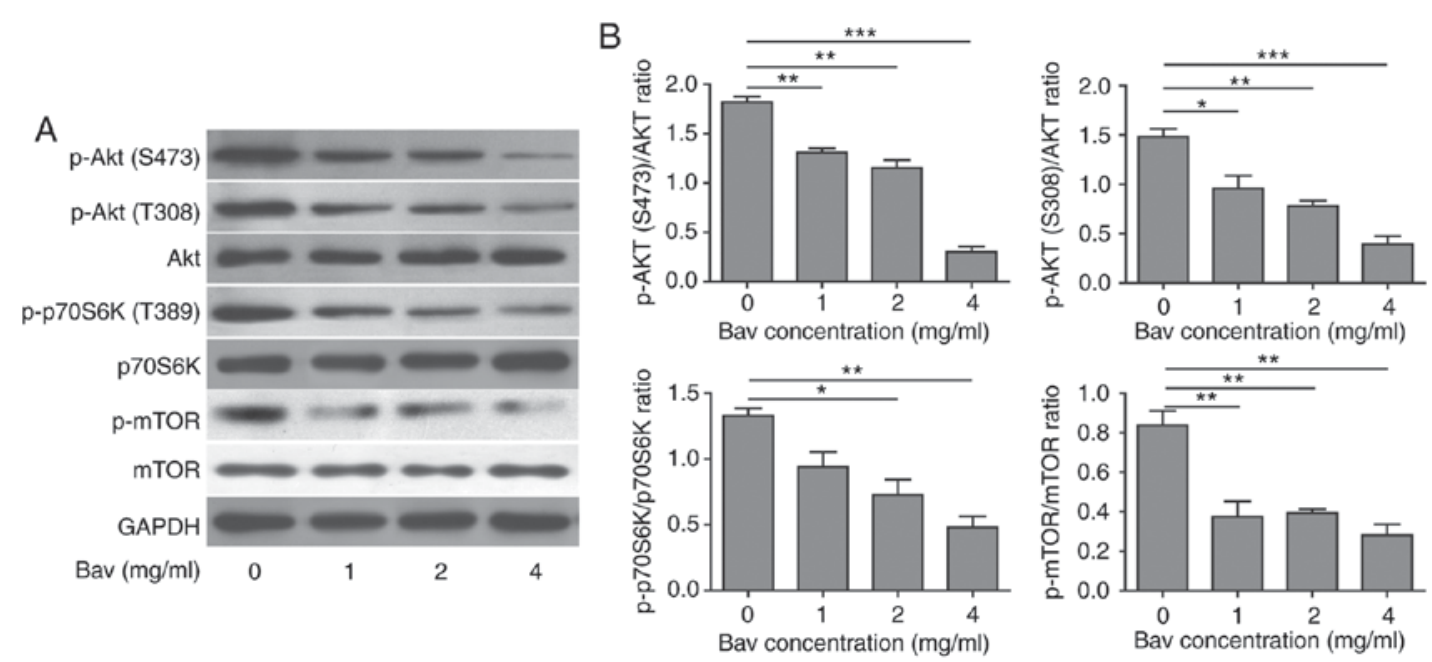

Figure 5. Bevacizumab can suppress the Akt-mTOR signaling pathway of glioblastoma cells to enhance their autophagy. (A) U87-MG cells were treated with different concentrations of bevacizumab for $48 \mathrm{~h}$, and then the cells were lysed for western blot analysis to assess the phosphorylation of indicative hallmarks in Akt-mTOR signaling pathway that involved in autophagy. GAPDH was used as the loading control. The result was a representative of three independent experiments. (B) The quantification of phosphorylated Akt (S473), Akt (T308), phosphorylated mTOR (S2448) and phosphorylated p70S6K those normalized to their corresponding total proteins, respectively, for result in A, all of them can be used to reflect the mTOR activity. Error bars represented mean \pm SD. P-values were determined by the one-way ANOVA followed by Tukey post hoc test. ${ }^{* * *} \mathrm{P}<0.001,{ }^{* *} \mathrm{P}<0.01$. ${ }^{*} \mathrm{P}<0.05$. ANOVA, analysis of variance.

with bevacizumab, but the underlying mechanism was still elusive. As is mentioned before, the autophagy process was found to be involved in the chemo-drug tolerance. To explore the relationship between bevacizumab-tolerance and autophagy, we used varying doses of bevacizumab to treat glioblastoma cells for $48 \mathrm{~h}$, and then assessed two hallmarks of autophagy, the LC3B-II/LC3B-I ratio and SQSTM1(p62) degradation by biochemical approaches. During autophagy, a cytosolic form of LC3B-I can be converted to a membrane-associated form LC3B-II by conjugating LC3B-I to phosphatidylethanolamine in the pre-autophagosomal and autophagosomal membranes through a ubiquitin like system (33). Thus, the relative contents of LC3B-II and the ratio of LC3B-II/LC3B-I were reliable indicators for monitoring the autophagy and autophagy-related processes in tumor cells. In addition, the level of SQSTM1 (p62) has also been used for monitoring autophagy, which is down-regulated when autophagy occurs (34). Meanwhile, SQSTM1 directly binds to LC3-II and mediates the targeted degradation of ubiquitinated protein aggregates (35). As is expected, glioblastoma cells showed gradually decreased LC3B-I, increased LC3B-II and thus upward LC3B-II/LC3B-I ratio as well as remarkable degradation of SQSTM1 (p62) upon bevacizumab treatment (Fig. 4). These results clearly proved that bevacizumab could enhance the autophagy of glioblastoma cells on a dose dependent manner, for which probably contributed to the formation of chemo-tolerance of glioblastoma cells.

Bevacizumab induced autophagy by suppressing Akt-mTOR signaling pathway. Previous studies have revealed that tumor cells could suppress Akt-mTOR signaling pathway to induce autophagy (36). In order to understand the detailed mechanism of enhanced autophagy in glioblastoma cells after bevacizumab treatment, we further performed some biochemical experiments to assess the Akt-mTOR signaling pathway by measuring the phosphorylation levels of Akt (T308 and S473), mTOR (S2448) and p70 ribosomal protein S6 kinase (p70S6K, Thr389). p70S6K is a direct substrate of mTOR and an established marker for mTOR signaling. Previous studies showed that mTOR was critical for autophagy induction, and the upstream PI3K-Akt signaling could activate mTOR thus suppressed autophagy, while repressed mTOR in the absence of growth factors could active autophagy (37). After treated with varying doses of 
A

A LC3B-I

SQSTM1

GAPDH

$\operatorname{Bav}(\mathrm{mg} / \mathrm{ml}) \quad-\quad+\quad+\quad-\quad+\quad+$

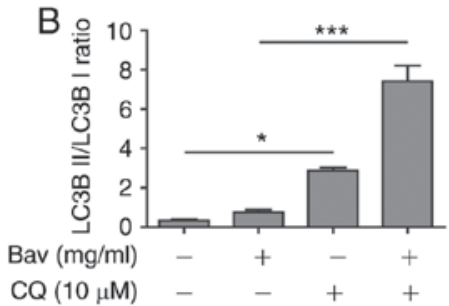

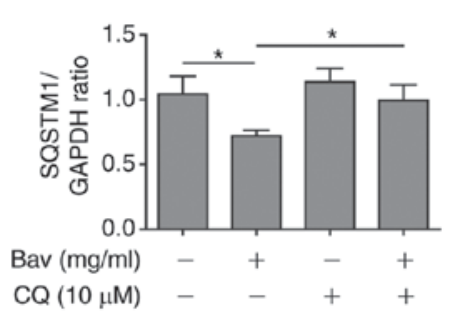

Figure 6. Chloroquine can significantly block the autophagy in glioblastoma cells induced by bevacizumab. (A) U87-MG cells were treated with 4 mg/ml bevacizumab in the presence or absence of $10 \mu \mathrm{M}$ chloroquine for $48 \mathrm{~h}$, and then the cells were lysed for western blot analysis for indicative hallmarks of autophagy. GAPDH was used as the loading control. The result was a representative of three independent experiments (B) The quantification of LC3B-II/LC3B-I ratio and SQSTM1(p62)/GAPDH ratio for result in A. Error bars represented mean \pm SD. P-values were determined by one-way ANOVA followed by Tukey's post hoc test. ${ }^{* * *} \mathrm{P}<0.001,{ }^{*} \mathrm{P}<0.05$. LC3, light chain 3; ANOVA, analysis of variance.
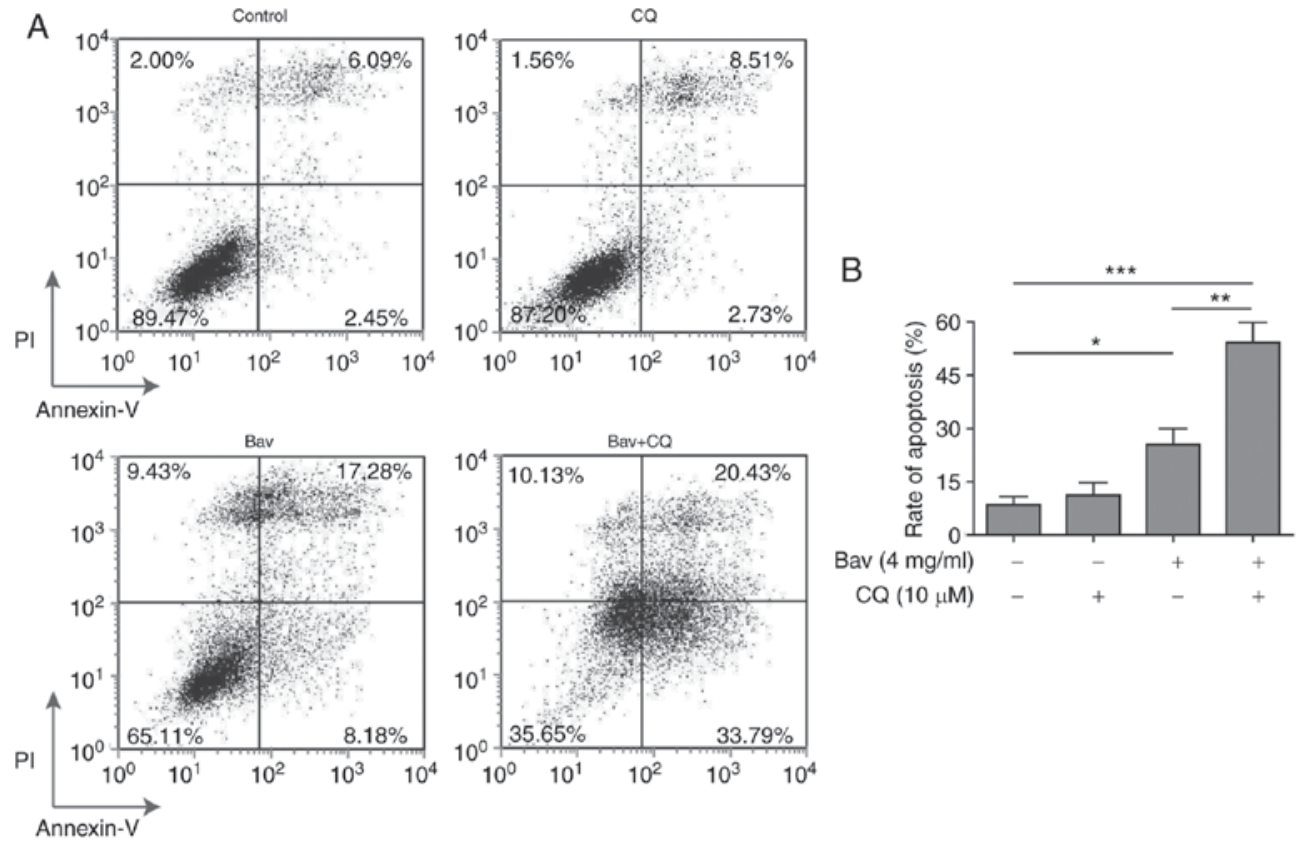

$\mathrm{CQ}(10 \mu \mathrm{M})$

Figure 7. Blocking the autophagy process can enhance the pro-apoptosis effect of bevacizumab on glioblastoma cells. U87-MG cells were treated with $4 \mathrm{mg} / \mathrm{ml}$ bevacizumab in the presence or absence of $10 \mu \mathrm{M}$ chloroquine for $48 \mathrm{~h}$, and then the cell apoptosis was assessed by Annexin V/PI staining and subsequent flow cytometry analysis. (A) One representative FACS result from three independent experiments. (B) The rate of apoptosis for cells in A. Error bars represented mean \pm SD. P-values were determined by one-way ANOVA followed by Tukey's post hoc test. ${ }^{* * *} \mathrm{P}<0.001,{ }^{* *} \mathrm{P}<0.01$, and ${ }^{*} \mathrm{P}<0.05$. ANOVA, analysis of variance.

bevacizumab for $48 \mathrm{~h}$, as expected, glioblastoma cells showed dramatically decreased phosphorylation levels of Akt, mTOR and p70S6K in a dose dependent manner (Fig. 5), which indicated that the Akt-mTOR signaling pathway was impaired. It has been proved that attenuated Akt-mTOR signaling pathway can result in autophagy (38). Based on our results, we concluded that bevacizumab could indeed suppress Akt-mTOR signaling pathway to induce high level of autophagy in glioblastoma cells, which was also consistent with previous results reported in other tumor cells (39).

Bevacizumab induced autophagy can be blocked by chloroquine in glioblastoma cells. In the later stage of autophagy, autophagosomes fuse with lysosomes to form autolysosomes, and the sequestered intra-autophagosomal components will be degraded by lysosomal hydrolases. Chloroquine (CQ), a well-known lysosome inhibitor, is therefore often used to inhibit autophagy process as it can accumulate in lysosome and raise the lysosomal $\mathrm{pH}$, which results in robust inhibition of lysosomal proteases that require an acidic $\mathrm{pH}$, the fusion of autophagosome with lysosome and lysosomal protein degradation (40). Here, we also treated glioblastoma cells with bevacizumab in the presence of $\mathrm{CQ}$, western blotting showed that sole bevacizumab treatment could upregulate the LC3B-II/LC3B-I ratio and downregulate SQSTM1 (p62) when compared with those without bevacizumab treatment (Fig. 6), which confirmed that autophagy was indeed induced by bevacizumab. Moreover, bevacizumab plus CQ further increased the levels of LC3-II and SQSTM1 when compared with those treated by bevacizumab alone (Fig. 6). The significantly accumulated LC3-II and SQSTM1 (p62) caused by inhibiting lysosomal protein degradation indicated that bevacizumab induced autophagy was severely blocked by CQ in glioblastoma cells.

Blocking the autophagy process enhanced the cytotoxicity of bevacizumab on glioblastoma cells. To verify whether bevacizumab resistance of glioblastoma cells was autophagy dependent, we further utilized CQ to inhibit the autophagy 
process to examine the apoptosis of glioblastoma cells under bevacizumab administration. As expected, the FACS results clearly demonstrated that combined treatment with bevacizumab and CQ displayed remarkably increased cytotoxicity on glioblastoma cells when compared to the sole treatment with either drug alone, from almost 54.22 to $25.46 \%$ (bevacizumab alone) or $11.24 \%$ (CQ alone) of cell apoptosis (Fig. 7). And the apoptosis result of bevacizumab treatment alone was consistent with that shown in Fig. 2. Meanwhile, the data in Fig. 2 demonstrated that $2 \mathrm{mg} / \mathrm{ml}$ and $4 \mathrm{mg} / \mathrm{ml}$ bevacizumab could cause distinct tolerance of glioblastoma cells, therefore, the augmented cell apoptosis resulted from the combination of CQ and the same dose of bevacizumab further proved that autophagy was a leading cause of drug-tolerance. Moreover, the tolerance of glioblastoma cells was relieved by blocking their autophagy process. As stated above, the tolerance occurred during bevacizumab administration was principally caused by autophagy induction in glioblastoma cells, and thus blocking the autophagy process was able to break the tolerance and enhance the cytotoxicity of bevacizumab.

\section{Discussion}

In recent clinical treatments, there are ever growing obstacles occurred in the therapy of glioblastoma patients, such as low efficacy, various side effects and knotty chemo-tolerance. All these problems lead to low cure rate, high mortality and high recurrence of glioblastoma. Despite we have made great progresses in developing new chemotherapeutic agents, chemo-tolerance is still the crucial issue for both clinicians and drug developers, which could severely dampen the efficacy of anticancer drugs in clinical application. To overcome this difficulty, many efforts have been put into the related study, however, the complexities of mechanisms of resistance that caused by tumor heterogeneity and microenvironment have seemingly hindered our steps to solve this problem.

In this study, we explored the effect of bevacizumab, one anti-angiogenic reagent, not a traditional chemo-drug, on the tolerance induction of glioblastoma from different angles. We firstly found that bevacizumab could directly suppress the proliferation of glioblastoma cells with a dose and time dependent manner. Meanwhile, bevacizumab was able to promote the apoptosis of tumor cells. The downregulated anti-apoptotic protein level and upregulated pro-apoptotic protein level synergistically lead to this striking effect of bevacizumab. Nevertheless, the apoptosis of glioblastoma cells reached a plateau under high dose of bevacizumab, which reflected that some glioblastoma cells could tolerate bevacizumab. The reasons behind this phenomenon were complex, and multiple changes were utilized by tumor cells to survive under metabolic stress in the microenvironment, which include the elevated autophagy. Interestingly, we also revealed that high dose of bevacizumab would induce the autophagy in glioblastoma cells, indicated by the increased LC3B-II/LC3B-I ratio and remarkable degradation of SQSTM1, which therefore counteracted the cytotoxicity of bevacizumab on tumor cells. In other related reports, hepatocellular carcinoma (HCC) cells treated with bevacizumab also showed inhibited cell growth, reduced new vessel developments and upregulated autophagy (41), which suggested that bevacizumab induced autophagy might be a general phenomenon in solid tumors.

Previous work has shown that autophagy can constrain tumor initiation in normal tissues by regulating DNA damage and oxidative stress. However, some established tumors also rely on autophagy for tumor promotion and maintenance (42). Mechanistic study further confirmed that bevacizumab treatment could induce autophagy of glioblastoma cells by suppressing the Akt-mTOR signaling pathway via reducing the phosphorylation of Akt, mTOR and mTOR's direct substrates p70S6K. Besides, AMPK signaling pathway was also shown to be involved in the autophagy induction (39). In other work, people also found that chemo-tolerance of non-small cell lung cancer could be contributed by autophagy in hypoxic conditions (43). Therefore, blocking autophagy process became an appealing tumor therapy and extensive biomedical studies were carried out to test the antitumor effect of various autophagy inhibitors (44-46). Furthermore, CQ was administered in combination with trastuzumab (Herceptin) in clinic to solve the drug tolerance in Her2 positive breast cancer patients, which successfully improved the efficacy of trastuzumab (27). In our study, we used CQ to block autophagy in glioblastoma cells. Significantly accumulated LC3B-II and SQSTM1 proteins clearly demonstrated that the autophagy process was severely blocked. Thus, we found the tumor-suppressive effect of bevacizumab was significantly enhanced, indicated by the remarkably increased tumor cell apoptosis after bevacizumab and CQ combination treatment. In some related reports, people also found that other autophagy inhibitors also had similar functions in different cancers $(44,45)$. Nevertheless, these encouraging improvements need further confirmation in human glioblastoma treatment when combined autophagy inhibitors with radiotherapy and chemotherapy. Moreover, the detailed mechanism of CQ on tumor cells may be complicated, not solely depending on the blocking of autophagy.

In summary, our study revealed that bevacizumab could induce the chemo-tolerance of glioblastoma cells by upregulating their autophagy level through inhibiting the Akt-mTOR signaling pathway. This novel mechanism will help us better understand the functional relevance of autophagy within the tumor microenvironment. Therefore, pharmacological or genetic inhibition of autophagy is a reasonable and promising way to enhance the efficacy of chemotherapy for glioblastoma and to improve clinical treatment of cancer patients. In future, more cooperation between laboratory and clinical research is still needed to design other therapeutic strategies to overcome the chemo-tolerance and to enhance the outcomes of anticancer therapies for cancer patients.

\section{References}

1. Omuro A and DeAngelis LM: Glioblastoma and other malignant gliomas: A clinical review. JAMA 310: 1842-1850, 2013.

2. Madany M, Thomas TM, Edwards L and Yu JS: Immunobiology and immunotherapeutic targeting of glioma stem cells. Adv Exp Med Biol 853: 139-166, 2015.

3. Kanu OO, Hughes B, Di C, Lin N, Fu J, Bigner DD, Yan H and Adamson C: Glioblastoma multiforme oncogenomics and signaling pathways. Clin Med Oncol 3: 39-52, 2009.

4. Tanaka S, Louis DN, Curry WT, Batchelor TT and Dietrich J: Diagnostic and therapeutic avenues for glioblastoma: No longer a dead end? Nat Rev Clin Oncol 10: 14-26, 2013. 
5. Zhang J, Stevens MF, Laughton CA, Madhusudan S and Bradshaw TD: Acquired resistance to temozolomide in glioma cell lines: Molecular mechanisms and potential translational applications. Oncology 78: 103-114, 2010.

6. Cahill DP, Levine KK, Betensky RA, Codd PJ, Romany CA, Reavie LB, Batchelor TT, Futreal PA, Stratton MR, Curry WT, et al: Loss of the mismatch repair protein MSH6 in human glioblastomas is associated with tumor progression during temozolomide treatment. Clin Cancer Res 13: 2038-2045, 2007.

7. Ramirez YP, Weatherbee JL, Wheelhouse RT and Ross AH: Glioblastoma multiforme therapy and mechanisms of resistance. Pharmaceuticals (Basel) 6: 1475-1506, 2013.

8. Chi AS, Sorensen AG, Jain RK and Batchelor TT: Angiogenesis as a therapeutic target in malignant gliomas. Oncologist 14: 621-636, 2009.

9. Das S and Marsden PA: Angiogenesis in Glioblastoma. N Engl J Med 369: 1561-1563, 2013

10. Wick W, Chinot OL, Bendszus M, Mason W, Henriksson R, Saran F, Nishikawa R, Revil C, Kerloeguen Y and Cloughesy T: Evaluation of pseudoprogression rates and tumor progression patterns in a phase III trial of bevacizumab plus radiotherapy/temozolomide for newly diagnosed glioblastoma. Neuro Oncol 18: 1434-1441, 2016.

11. Chinot OL, Wick W, Mason W, Henriksson R, Saran F, Nishikawa R, Carpentier AF, Hoang-Xuan K, Kavan P, Cernea D, et al: Bevacizumab plus radiotherapy-temozolomide for newly diagnosed glioblastoma. N Engl J Med 370: 709-722, 2014.

12. Jahangiri A, De Lay M, Miller LM, Carbonell WS, Hu YL, Lu K, Tom MW, Paquette J, Tokuyasu TA, Tsao S, et al: Gene expression profile identifies tyrosine kinase c-Met as a targetable mediator of antiangiogenic therapy resistance. Clin Cancer Res 19: 1773-1783, 2013.

13. Piao Y, Liang J, Holmes L, Zurita AJ, Henry V, Heymach JV and de Groot JF: Glioblastoma resistance to anti-VEGF therapy is associated with myeloid cell infiltration, stem cell accumulation, and a mesenchymal phenotype. Neuro Oncol 14: 1379-1392, 2012.

14. Mesange P, Poindessous V, Sabbah M, Escargueil AE, de Gramont A and Larsen AK: Intrinsic bevacizumab resistance is associated with prolonged activation of autocrine VEGF signaling and hypoxia tolerance in colorectal cancer cells and can be overcome by nintedanib, a small molecule angiokinase inhibitor. Oncotarget 5: 4709-4721, 2014.

15. Wileman T: Aggresomes and autophagy generate sites for virus replication. Science 312: 875-878, 2006

16. Münz C: Autophagy and antigen presentation. Cell Microbiol 8: 891-898, 2006

17. Levine B and Kroemer G: Autophagy in the pathogenesis of disease. Cell 132: 27-42, 2008

18. Mizushima N, Levine B, Cuervo AM and Klionsky DJ: Autophagy fights disease through cellular self-digestion. Nature 451: 1069-1075, 2008

19. Choi AM, Ryter SW and Levine B: Autophagy in human health and disease. N Engl J Med 368: 651-662, 2013.

20. Murrow L and Debnath J: Autophagy as a stress-response and quality-control mechanism: Implications for cell injury and human disease. Annu Rev Pathol 8: 105-137, 2013.

21. Moscat J and Diaz-Meco MT: p62 at the crossroads of autophagy, apoptosis, and cancer. Cell 137: 1001-1004, 2009.

22. Gugnoni M, Sancisi V, Manzotti G, Gandolfi G and Ciarrocchi A: Autophagy and epithelial-mesenchymal transition: An intricate interplay in cancer. Cell Death Dis 7: e2520, 2016.

23. Zhong Z, Sanchez-Lopez E and Karin M: Autophagy, inflammation and immunity: A troika governing cancer and its treatment. Cell 166: 288-298, 2016.

24. Dou Z, Xu C, Donahue G, Shimi T, Pan JA, Zhu J, Ivanov A, Capell BC, Drake AM, Shah PP,et al: Autophagy mediates degradation of nuclear lamina. Nature 527: 105-109, 2015.

25. Perera RM, Stoykova S, Nicolay BN, Ross KN, Fitamant J, Boukhali M, Lengrand J, Deshpande V, Selig MK Ferrone CR, et al: Transcriptional control of autophagy-lysosome function drives pancreatic cancer metabolism. Nature 524: 361-365, 2015

26. Sui X, Chen R, Wang Z, Huang Z, Kong N, Zhang M, Han W, Lou F, Yang J, Zhang Q, et al: Autophagy and chemotherapy resistance: A promising therapeutic target for cancer treatment. Cell Death Dis 4: e838, 2013.
27. Cufi S, Vazquez-Martin A, Oliveras-Ferraros C, Corominas-Faja $\mathrm{B}$, Cuyàs $\mathrm{E}$, López-Bonet $\mathrm{E}$, Martin-Castillo $\mathrm{B}$, Joven J and Menendez JA: The anti-malarial chloroquine overcomes primary resistance and restores sensitivity to trastuzumab in HER2-positive breast cancer. Sci Rep 3: 2469, 2013.

28. Tang MC, Wu MY, Hwang MH, Chang YT, Huang HJ, Lin AM and Yang JC: Chloroquine enhances gefitinib cytotoxicity in gefitinib-resistant nonsmall cell lung cancer cells. PLoS One 10: $\mathrm{e} 0119135,2015$.

29. Aveic S and Tonini GP: Resistance to receptor tyrosine kinase inhibitors in solid tumors: Can we improve the cancer fighting strategy by blocking autophagy? Cancer Cell Int 16: 62, 2016.

30. Allen M, Bjerke M, Edlund H, Nelander S and Westermark B: Origin of the U87MG glioma cell line: Good news and bad news. Sci Transl Med 8: 354re3, 2016.

31. Dolgin E: Venerable brain-cancer cell line faces identity crisis Nature 537: 149-150, 2016.

32. Han J, Jun Y, Kim SH, Hoang HH, Jung Y, Kim S, Kim J, Austin RH, Lee S and Park S: Rapid emergence and mechanisms of resistance by U87 glioblastoma cells to doxorubicin in an in vitro tumor microfluidic ecology. Proc Natl Acad Sci USA 113: 14283-14288, 2016.

33. Ichimura Y, Kirisako T, Takao T, Satomi Y, Shimonishi Y, Ishihara N, Mizushima N, Tanida I, Kominami E, Ohsumi M, et al: A ubiquitin-like system mediates protein lipidation. Nature 408: 488-492, 2000.

34. Larsen KB, Lamark T, Øvervatn A, Harneshaug I, Johansen T and Bjørkøy G: A reporter cell system to monitor autophagy based on p62/SQSTM1. Autophagy 6: 784-793, 2010.

35. Johansen T and Lamark T: Selective autophagy mediated by autophagic adapter proteins. Autophagy 7: 279-296, 2011

36. Ravikumar B, Sarkar S, Davies JE, Futter M, Garcia-Arencibia M, Green-Thompson ZW, Jimenez-Sanchez M, Korolchuk VI, Lichtenberg M,Luo S, et al: Regulation of mammalian autophagy in physiology and pathophysiology. Physiol Rev 90: 1383-1435, 2010.

37. Schmelzle T and Hall MN: TOR, a central controller of cell growth. Cell 103: 253-262, 2000.

38. Shao X, Lai D, Zhang L and Xu H: Induction of autophagy and apoptosis via PI3K/AKT/TOR pathways by azadirachtin a in Spodoptera litura cells. Sci Rep 6: 35482, 2016.

39. Zha QB, Zhang XY, Lin QR, Xu LH, Zhao GX, Pan H, Zhou D, Ouyang DY, Liu ZH and He XH: Cucurbitacin E induces autophagy via downregulating mTORC1 signaling and upregulating AMPK activity. PLoS One 10: e0124355, 2015.

40. Shintani T and Klionsky DJ: Autophagy in health and disease: A double-edged sword. Science 306: 990-995, 2004.

41. Guo XL, Li D, Sun K, Wang J, Liu Y, Song JR, Zhao QD, Zhang SS, Deng WJ, Zhao X, et al: Inhibition of autophagy enhances anticancer effects of bevacizumab in hepatocarcinoma. J Mol Med (Berl) 91: 473-483, 2013.

42. White E and DiPaola RS: The double-edged sword of autophagy modulation in cancer. Clin Cancer Res 15: 5308-5316, 2009.

43. Lee JG, Shin JH, Shim HS, Lee CY, Kim DJ, Kim YS and Chung KY: Autophagy contributes to the chemo-resistance of non-small cell lung cancer in hypoxic conditions. Respir Res 16: 138,2015

44. Dragowska WH, Weppler SA, Wang JC, Wong LY, Kapanen AI, Rawji JS, Warburton C, Qadir MA, Donohue E, Roberge M, et al: Induction of autophagy is an early response to gefitinib and a potential therapeutic target in breast cancer. PLoS One 8: e76503, 2013.

45. Yang Z, Liu Y, Wei X, Zhou X, Gong C, Zhang T, Jin P, Xu S, Ma D and Gao Q: Co-targeting EGFR and autophagy impairs ovarian cancer cell survival during detachment from the ECM. Curr Cancer Drug Targets 15: 215-226, 2015.

46. Nihira K, Miki Y, Iida S, Narumi S, Ono K, Iwabuchi E, Ise K, Mori K, Saito M, Ebina M, et al: An activation of LC3A-mediated autophagy contributes to de novo and acquired resistance to EGFR tyrosine kinase inhibitors in lung adenocarcinoma. J Pathol 234: 277-288, 2014.

This work is licensed under a Creative Commons Attribution-NonCommercial-NoDerivatives 4.0 International (CC BY-NC-ND 4.0) License. 